\title{
Innovation Strategies for Combating Occupational Stress and Fatigue in Medical Imaging
}

\author{
Bruce I. Reiner • Elizabeth Krupinski
}

Published online: 6 December 2011

(C) Society for Imaging Informatics in Medicine 2011

\section{Introduction}

The adverse effect of occupational stress and fatigue on job performance was first documented by Lazarus et al. in 1952 [1]. Later work by Cohen in 1980 [2] reported occupational stress to be a source of cognitive fatigue and diminished energy, leading to information overload. While this earlier work was not directly related to healthcare, many observations are relevant to contemporary medical practice. In addition to cognitive fatigue and information overload, a variety of occupational stressors have been described including bureaucratic frustration and task load, which lead to diminished job performance and perceptual distractions [2]. It is important to note that the incidence and severity of occupational stress and fatigue is highly variable and dependent upon a number of individual worker attributes, including personality, job experience, age, and fear of negative evaluation $[3,4]$.

The ubiquitous nature of occupational stress and fatigue in medicine has been well described and reported to serve as a major source of medical errors [5-8]. Medical imaging is especially prone to occupational stress and fatigue due to increasing workload volume and complexity, heightened

B. I. Reiner $(\square)$

Department of Radiology,

Veterans Affairs Maryland Healthcare System,

10 North Greene Street,

Baltimore, MD 21201, USA

e-mail: breiner1@comcast.net

E. Krupinski

Department of Radiology, Psychology and the Arizona

Telemedicine Program, University of Arizona,

1609 N Warren, Bldg 211, Rm 112,

Tucson, AZ 85724, USA

service demands, and dependence upon computerized technologies $[9,10]$. In spite of these well-documented concerns regarding occupational stress and fatigue in medical practice, surprisingly little is currently being done to proactively intervene. Most existing stress management interventions are "after the fact" and often triggered by an adverse clinical event. A unique opportunity exists for healthcare professionals and technology providers to study the causes of occupational stress and fatigue, objectively analyze its effects on job performance, and develop interventions at the point of care.

\section{Existing Strategies for Occupational Stress Management}

In an attempt to manage stress in the workplace, many organizations have adopted stress management programs which can target any one of three different points in the stress cycle which include the intensity of stressors, the employee's appraisal of stress, or the employee's ability to cope with the outcomes [11]. Existing stress management interventions (SMIs) can be classified as primary, secondary, or tertiary [12]. Primary SMIs attempt to alter the sources of stress (e.g., workflow redesign), secondary SMIs attempt to reduce stress severity (e.g., mandatory breaks), while tertiary SMIs are designed to treat stress through mental health programs.

The most common SMIs in current use are secondary programs aimed at moderating the individual's stress response and include cognitive behavior skills training, meditation, relaxation techniques, and exercise [13]. Another less commonly used type of SMI is journaling; which requires the employee to maintain a journal, log, or diary of the stressful events [14]. The purpose of journaling is to provide a personalized means of monitoring stress, identify causative factors, and measure response to different interventions. 
While a number of technologies currently exist to measure visual fatigue and physiologic stress [15], direct integration into imaging and information system technologies is not currently available. As a result, measures of occupational stress and fatigue among medical imaging professionals are largely limited to subjective and intrapersonal evaluation, which is not reproducible and difficult to quantify. Table 1 lists a number of deficiencies with existing occupational SMIs, which ultimately limit their effectiveness in current healthcare practice. In order to understand the incidence and severity of occupational stress and fatigue, it is essential that a methodology for prospective, objective, and standardized data analysis be created which can take into account contributing factors such as technology in use, end-user characteristics, and task complexity. The derived data analytics should ideally be delivered at the point of care, customized to the specific needs and preferences of the individual end-user, and take into account historical context and user-specific data to predict future stress/fatigue variations, along with appropriate interventions.

One industry where technology has been creatively used for fatigue detection and intervention is transportation, which currently utilizes vision-based facial expression recognition to prospectively detect driver fatigue $[16,17]$. In addition to hardware technologies used to detect driver fatigue in real time, software technologies are also utilized to predict future operator fatigue based upon historical data analysis [18]. This two-pronged approach of technologyderived fatigue detection and prediction can serve as an innovation model for SMI in medicine.

\section{Innovation Strategy}

An optimal SMI innovation strategy would in effect address all of the deficiencies outlined in Table 1 while adhering to the following five major innovation objectives:

1. Customization

2. Adaptability
3. Interactive

4. Data-driven

5. Quality-centric

Customization requires configuring the technology in accordance with the individual end-user's fatigue profile and preferences [19]. In addition to the technology maintaining flexibility to the individual end-user, it should also be adaptive relative to the task being performed [20]. This task adaptability may take a number of forms, including technology modification relative to workload volume, task complexity, technology in use, and performance deliverables. Computer-human interaction would be customizable in keeping with each individual end-user's preferences and could even be incorporated into a video game format for improved acceptance [21]. In order to ensure that the fatigue data and interventions ultimately translate into improve performance measures, the data analytics would include longitudinal correlation of stress/fatigue and quality data.

In a manner similar to existing models of analog journaling, the proposed technology offers the opportunity to perform prospective computerized journaling through the integration of electronic auditing tools into the technologies being used [22]. As individual workflow steps are electronically captured, simultaneous fatigue measures are recorded in real time. This provides an objective and reproducible means of determining the relationship between user and context-specific workflow, stress, and technology. Those end-users found to exhibit higher than expected occupational stress/fatigue levels may benefit from the experience of similar profile peers. Comparative analysis of workflow provides unique educational and training opportunities for "high stress" end-users to mimic the workflow of their "low stress" colleagues.

During the course of everyday workflow, the fatigue tracking and analytical tools can provide both real-time and predictive measurements of fatigue. Predictive fatigue measures are the compilation of recent trending analysis, the individual end-user's historical profile, the tasks being performed, and the technology in use. As actual or predicted

Table 1 Existing deficiencies of occupational SMIs

1 Stress/fatigue analysis is largely subjective in nature.

2 Lack of supporting technology for real-time fatigue/stress measurements.

3 Minimal correlation of reported stress/fatigue measures with performance outcomes.

4 Recorded data are non-standardized, limiting meta-analysis across multiple sites.

5 Interventions tend to be systematic in nature and not customized to the specific needs and preferences of individual workers.

6 Contributing role of technology is often understated and/or misunderstood.

7 Data analyses are often retrospective and not prospective in nature.

8 Task performance is typically analyzed collectively so that individual task complexity (i.e., context specificity) is often missing from the analysis.

9 Analyses often limit contributing role of user-specific attributes (e.g., personality, experience).

10 Feedback and education is typically "after the fact" and not delivered at the point of care (where interaction effect can be maximized). 
fatigue measures exceed a predetermined threshold, an automated alert or prompt could be sent to the end-user notifying them of the fatigue concern. The computer could then provide the end-user with a number of potential intervention options, in keeping with the individual end-user's profile, defined preferences, departmental policies, workflow options, and staff availability. In addition to traditional interventions such as a break from work and deferral to another staff member, creative interventions can be proposed such as "reduced stress workflow." In this option, the worker would continue to perform their duties but do so under modified working conditions aimed at reducing stress/fatigue. This may consist of adjusting work assignments to tasks of lesser complexity or utilizing alternative workflow techniques aimed at reducing fatigue.

In the example of a radiologist tasked with the interpretation of a chest CT angiogram for evaluation of pulmonary embolism, a number of potential workflow modifications may be considered for stress/fatigue reduction by reducing cognitive overload. Examples of potential workflow modifications may include the following:

1. Preliminary interpretation using computer-aided diagnosis software

2. Automated extraction of historical imaging/report data

3. Modification of the user interface (e.g., simplification of the tool bar)

4. Auto-population of previous report findings onto the current report template [23]

5. Implementation of a context-specific automated workflow template [24]

6. Customized presentation of image reconstructions

Understanding the complex relationship between occupational stress/fatigue and quality of performance is a fundamental goal of the proposed technology, which is not present in existing SMIs. A number of quality metrics can be incorporated into the stress/fatigue database (Table 2), which can be used in the assessment of visual, physiologic, and cognitive forms of fatigue [15]. In addition to elucidating the relationship between fatigue and performance, these data

Table 2 Quality metrics contained within the stress/fatigue database

\begin{tabular}{ll}
\hline 1 & Eye tracking efficiency \\
2 & Data accessibility and review \\
3 & Report quality and timeliness \\
4 & Diagnostic accuracy \\
5 & Exam and protocol optimization \\
6 & Image quality \\
7 & Iatrogenic complications and documentation \\
8 & Compliance with standards and regulations \\
9 & Communication and consultation \\
10 & Technology utilization \\
\hline
\end{tabular}

can also be used in the creation of best practice guidelines, education/training tools, and workflow optimization. By identifying the most efficient end-users within different profile groups (as defined by the highest quality scores and lowest fatigue measures), it is possible to determine the specific tools, educational resources, workflow patterns, and data extraction/ presentation techniques most beneficial in everyday practice. In addition to the evaluation of human performance, the database can also be of value in comparative technology assessment. As technology vendors are provided with objective data related to context- and user-specific fatigue and quality measures, valuable insights can be gained in targeting R\&D efforts, product refinement, and new technology development.

While understanding the causative relationship and interaction effects between occupational stress/fatigue, technology usage, and quality performance is the primary objective of the proposed technology, a secondary goal is the optimization and customization of stress interventions. A number of intervention options are readily available (Table 3 ), which can be directly integrated into the workstation and immediate environment. These interventions can be customized to the specific needs and preferences of each individual end-user, with data-derived feedback provided to each end-user on the relative success under different circumstances. An end-user could elect to query the stress/fatigue database for recommendations based upon the objective analysis and collective experience of end-users with similar profiles and tasks being performed. The goal is to leverage the standardized data contained within the database for proactive intervention, which can be customized and adapted to the unique needs of each individual end-user, while providing education and training aids for improved performance.

\section{Conclusion}

While occupational stress and fatigue have been well described in medicine and shown to have an adverse effect on

Table 3 Options for stress/fatigue intervention

$\begin{array}{ll}1 & \text { Environmental (temperature, light, ventilation) } \\ 2 & \text { Exercise (ocular, aerobic, anaerobic) } \\ 3 & \begin{array}{c}\text { Relaxation techniques (stretching, breathing, yoga, nap, } \\ \text { medication) }\end{array} \\ 4 & \text { Dietary (herbal supplements, pharmacologic agents) } \\ 5 & \text { Massage } \\ 6 & \text { Aromatherapy } \\ 7 & \text { Online games and puzzles } \\ 8 & \text { Music } \\ 9 & \text { Laughter } \\ 10 & \text { Imagery }\end{array}$


patient safety and quality, existing interventional techniques are insufficient and lack objective data analysis. Through the direct integration of stress and fatigue measurement tools into the modality and information systems workstations, a methodology can be created to objectively measure stress and fatigue in real time while providing direct feedback at the point of care. The derived data can in turn be used in the creation of user- and context-specific stress/ fatigue databases, which can take into account the unique attributes of individual end-users, technology in use, and tasks being performed. These data can in turn be correlated with a number of quality performance metrics in order to understand the causative and interaction effects between occupation stress/fatigue and performance. The database can also be used in the creation and assessment of customizable stress intervention techniques, education and training, workflow optimization, and best practice guidelines. While technology can be contributory to occupational stress and fatigue, it can also serve as part of the solution through datadriven innovation and adaptive customization.

\section{References}

1. Lazarus RS, Deese J, Osler JF: The effects of psychological stress upon performance. Psychol Bull 49:293-316, 1952

2. Cohen S: After effects of stress on human performance and social behavior: a review of research and theory. Psychol Bull 88:82108, 1980

3. Beehr TA, Newman JE: Job stress, employee health, and organizational effectiveness: a facet analysis, model and literature review. Personnel Psychology 31:665-699, 1978

4. Ivancevich JM, Matteson MT: A type A-B person-work environment interaction model for examining occupational stress and consequences. Human Relations 37:491-513, 1984

5. Leape LL: Errors in medicine. JAMA 272:1851-1857, 1994

6. Kohn LT, Corrigan J, Donaldson MS Eds. To err is human: building a safer health system. Washington DC: National Academy Press; 2000.

7. Helmreich RL: On error management: lessons from aviation. BMJ $32: 781-785,2000$
8. Reiner B, Krupinski E: The insidious problem of fatigue in medical imaging practice. J Digit Imaging. doi:10.1007/s10278-011-9436-4 (in press)

9. Sunshine JH, Maynard CD: Update on the diagnostic radiology employment market: findings through 2007-2008. J Am Coll Radiol 2008; 5:827-833 (and additional references)

10. Krupinski EA, Berbaum KS, Caldwell RT, et al: Long radiology workdays reduce detection and accommodation accuracy. J Am Coll Radiol 7:698-704, 2010

11. Ivancevich JM, Matteson MT, Freedman SM, et al: Worksite stress management interventions. Am Psychol 45:252-261, 1990

12. Richardson KM, Rothstein HR: Effects of occupational stress management intervention programs: a meta-analysis. J Occup Health Psychol 13:69-93, 2008

13. Giga SI, Cooper CL, Faragher B: The development of a framework for a comprehensive approach to stress management interventions at work. International Journal of Stress Management 10:280-296, 2003

14. Alford WK, Malouff JM, Osland KS: Written emotional expression as a coping method in child protective services. Journal of Stress Management 12:177-187, 2005

15. Reiner B, Krupinski E: Real-time occupational stress and fatigue measurement in medical imaging practice. J Digit Imaging. doi:10.1007/s10278-011-9439-1 (in press)

16. Senaratne R, Jap B, Lal S, et al: Comparing two video-based techniques for driver fatigue detection: classification versus optical flow approach. Machine Vision and Applications 22:597-618, 2011

17. Zhang Z, Zhang J: A new real-time eye tracking based on nonlinear unscented Kalman filter for monitoring driver fatigue. J Control Theory Appl 8:181-188, 2010

18. Horberry T, Hartley L, Krueger G et al.: Fatigue detection technologies for drivers: a review of existing operator-centered systems. Human Interfaces in Control Rooms, Cockpits, and Command Centers 2001. People in Control. The Second International Conference on (IEEE Conf Publication No. 481), 321-326

19. Reiner B, Krupinski E: Demystifying occupational stress and fatigue through the creation of an adaptive end-user profiling system. J Digit Imaging. doi:10.1007/s10278-011-9441-7 (in press)

20. Reiner B: One size (doesn't) fit all. J Am Coll Radiol 4:567-570, 2008

21. Reiner B, Siegel E: The potential for gaming techniques in radiology education and practice. J Am Coll Radiol 2:110-114, 2008

22. Reiner B: Automating radiologist workflow. Part II: hands free navigation. J Am Coll Radiol 11:1137-1141, 2008

23. Reiner BI: Medical imaging data reconciliation. Part 3: reconciliation of historical and current radiology report data. J Am Coll Radiol 8:768-771, 2011

24. Reiner B: New strategies for medical data mining. Part 3: automated workflow and optimization. J Am Coll Radiol 8:132-138, 2011 